\title{
Des expériences du surnaturel
}

Argument

Jean-Pierre Albert et Guillaume Rozenberg

\section{(2) OpenEdition}

Journals

Édition électronique

URL : http://journals.openedition.org/assr/20977

DOI : $10.4000 /$ assr. 20977

ISSN : 1777-5825

Éditeur

Éditions de l'EHESS

Édition imprimée

Date de publication : 1 janvier 2009

Pagination : 9-14

ISBN : 978-2-7132-2215-3

ISSN : 0335-5985

Référence électronique

Jean-Pierre Albert et Guillaume Rozenberg, « Des expériences du surnaturel », Archives de sciences sociales des religions [En ligne], 145 I janvier-mars 2009, mis en ligne le 01 janvier 2012, consulté le 01 mai 2019. URL : http://journals.openedition.org/assr/20977 ; DOI : 10.4000/assr.20977 


\section{Jean-Pierre Albert, Guillaume Rozenberg* Des expériences du surnaturel Argument}

Juillet 1882. Le Colonel américain H.S. Olcott, cofondateur de la Société théosophique et premier Occidental des temps modernes converti au bouddhisme, embarque pour Ceylan. Ce sera son troisième séjour sur l'île en deux ans. Dans le sillage du mouvement cinghalais de réveil religieux, qui répond à l'intense prosélytisme des missionnaires chrétiens favorisé par le règne britannique, le personnage a entrepris de réformer le bouddhisme local. Pétri du préjugé orientaliste qui voit dans les sociétés bouddhiques contemporaines tous les symptômes d'une dégénérescence religieuse, il appelle au retour à la lettre de l'enseignement du Bouddha - telle qu'elle est comprise, alors, en Occident. Il s'agit de ré-instiller, dans la population, la substance d'une tradition spirituelle que le temps et des penchants trop humains ont pervertie en dévotion superstitieuse. Lors de ses deux précédents voyages, le Colonel, monté sur un char à bœufs de sa confection, a parcouru l'île pour délivrer des conférences et répandre ses idées ; il a stimulé la création d'écoles qui font concurrence aux institutions chrétiennes. Le catéchisme bouddhique qu'il a rédigé afin d'instruire les Cinghalais dans leur propre religion a reçu l'imprimatur d'un moine des plus en vue ; l'ouvrage connaîtra une postérité sans pareille. Bref, Olcott, porté par l'aura que lui confère sa qualité de bouddhiste blanc, semble en passe de réussir son pari.

Or, à son arrivée à Ceylan, force lui est de déchanter. La branche bouddhique de la Société théosophique qu'il vient d'inaugurer est déjà moribonde, et le fonds mis en place pour financer le développement d'un système d'éducation vernaculaire n'a pas recueilli un centième des dons espérés. Pire, le christianisme a gagné du terrain. Par l'œuvre de missionnaires catholiques, un puits situé à proximité d'un lieu de pèlerinage bouddhique est devenu le théâtre de cures miraculeuses. Olcott n'en peut mais, le voici, au titre de la défense de la religion bouddhique, entraîné dans la course à l'extraordinaire. Quoique son catéchisme professe une antinomie

* Ce numéro thématique est le résultat de deux ateliers, organisés en juin 2007 (Paris) et février 2008 (Toulouse), autour des expériences du surnaturel. Nous tenons à remercier l'ensemble des participants à ces journées, y compris Sébastien Tank qui s'est joint à la discussion des différents textes lors de la seconde rencontre.

ARCHIVES DE SCIENCES SOCIALES DES RELIGIONS 145 (janvier-mars 2009), pp. 9-14 
du bouddhisme avec le miraculeux, le personnage se mue en thaumaturge. Un paralytique sur qui, au nom du Bouddha, il appose les mains retrouve ses capacités motrices. Le succès est instantané. Le séjour sur l'île, entamé sous de sombres auspices, se termine en tournée triomphale, durant laquelle le Colonel fait usage et étalage de ses pouvoirs thérapeutiques ${ }^{1}$.

Les tribulations cinghalaises du Colonel Olcott, en mettant en évidence l'impératif miraculeux d'une religion supposée rationnelle et sans dieu, illustrent la proposition première dont ce numéro thématique se soutient : le surnaturel, soit un type de phénomènes distingués des phénomènes normaux, impliquant l'idée d'entités ou de forces invisibles en mesure d'influer sur le cours du monde et des existences, entre toujours dans la définition d'une religion (à condition, bien entendu, d'ignorer les "religions séculières", telles les cultes à la nation). Pour le dire simplement : il n'y a religion que s'il y a extraordinaire. La proposition, des plus anciennes et des plus banales, n'a jamais fait l'unanimité. Émile Durkheim, dans Les Formes élémentaires de la vie religieuse, s'employait déjà à la réfuter. Nonobstant, la conviction d'une affinité substantielle entre le surnaturel et la religion perdure, et ce, non sans raison, renforcée qu'elle est par la perspective d'une théorie de la connaissance - l'anthropologie cognitive - différente, car plus psychologique que sociologique, de celle qu'envisageait Durkheim ${ }^{2}$.

Encore convient-il de lever une ambiguiité : recourir au vocable de « surnaturel » ne revient pas à endosser le soi-disant préjugé occidental, que tant d'observations ethnographiques infirment, d'une séparation nette entre deux sphères, la nature et la surnature. Le surnaturel, au sens où nous l'entendons, ne s'oppose pas à la nature, où il se déploie. Il s'oppose plutôt au naturel, considéré comme l'ensemble des phénomènes qui n'appellent de la part des membres d'une communauté aucune interrogation en termes d'étrangeté ou d'illisibilité. Théorisé ou non, le surnaturel est senti (pour parler comme Lucien Lévy-Bruhl) sur un mode particulier par les individus; son domaine est, d'une façon ou d'une autre, spécifié. S'il n'y a pas rupture de plan, il y a, à tout le moins, ligne de partage. Une ligne de partage dont l'accentuation et le tracé varient en fonction des cultures. L'ethnographie de la religion a pour tâche, entre autres, d'appréhender la démarcation entre pensée du naturel et pensée du surnaturel au sein d'une société, que la discrimination s'exprime sur un mode affectif, intellectuel ou rituel.

Il est un genre d'événements où se mettent en branle, et donc s'offrent à étudier, les mécanismes de cette discrimination : les « expériences du surnaturel ». Quelles que soient ses modalités d'expression, le surnaturel, en même temps qu'il est conçu comme mystérieux, est dit relever de l'expérience, faire l'objet d'une perception, d'un contact, d'un ressenti, d'une connaissance. Quoique situé en

1. Cf. Stephen Prothero, The White Buddhist. The Asian Odyssey of Henry Steel Olcott, Delhi, Sri Satguru Publications, 1997 [1996], pp. 85-115.

2. Voir, dans ce même numéro, la postface de Jean-Pierre Albert, lequel, en retour des contributions, reconsidère la notion de "surnaturel ». 
décalage avec l'ordinaire et le tangible, le surnaturel reste vécu. Ce vécu partagé diffère de l'expérience normale, parce que teinté d'indétermination : son référent (une divinité, un génie, un esprit de mort, etc.) et le moyen par lequel il est censé se manifester (mysticisme, chamanisme, possession, apparition, etc.) ne présentent pas un caractère transparent ou évident. La signification et la validité d'une expérience du surnaturel lui viennent en fait, non de son impossible positivité, mais de sa facticité : une machinerie sociale complexe forge le phénomène de toutes pièces. Une expérience du surnaturel prend forme et réalité sous l'effet d'un faisceau de représentations collectives, d'une toile de relations humaines, d'une mise en scène, d'une gestuelle, de discours, ainsi que par la manière dont se définissent et se formulent, en référence à l'événement, des façons de penser et d'agir. Elle existe par élaboration commune, elle est la constitution et l'interprétation d'une situation par un groupe d'individus en interaction. Les uns participent à sa réalisation en tant qu'officiants, les autres en tant que bénéficiaires, instigateurs, spectateurs, témoins, glossateurs, censeurs. En un mot, les expériences du surnaturel sont produites plutôt qu'elles ne se produisent. D'où la proposition suivante qui sous-tend ce dossier, condition nécessaire à une enquête des sciences sociales sur le surnaturel : les expériences du surnaturel ont cette particularité d'être représentées comme immédiates et d'être néanmoins toujours médiatisées.

L'optique qui est la nôtre s'écarte ainsi du projet d'un William James ou d'un Rudolf Otto, lesquels s'attachent à identifier des états psychologiques caractéristiques de l'expérience religieuse (ou expérience du sacré), une expérience supposée parler d'elle-même en ce qu'elle ressortirait à l'ordre, naturel plutôt que social, des instincts ou des sentiments. Au vrai, les expériences du surnaturel tendent parfois, à leur genèse présumée, vers le degré zéro de l'empirique et du ressenti, vers une absence de vécu subjectif. Les esprits, aux yeux des Kulung du Népal, se manifestent en règle générale sans rien laisser deviner, en provoquant une maladie dont l'origine est établie a posteriori, après l'échec des soins habituels ; les rares fois où ils se donnent à voir, leur perception est tronquée et incertaine, douteuse. L'objectivation de ces entités fuyantes exige un laborieux travail de la communauté, manufacture de l'expérience.

Aussi les expériences du surnaturel, problématiques par essence, soulèventelles la question de la croyance (au sens d'adhésion à un phénomène susceptible de doute), de ses ressorts et de ses modalités. Le Colonel Olcott, à la vérité, ne croyait pas au caractère surnaturel des guérisons qu'il accomplissait. Loin des oreilles de ses zélateurs, il invoquait la doctrine magnétique du médecin Franz Mesmer (1734-1815) avec laquelle il s'était familiarisé dans sa jeunesse et qui, à l'instant de se jeter dans la bataille du surnaturel contre les missionnaires, inspira son style thérapeutique. Il expliquait les rétablissements fulgurants dont il était responsable par une circulation de fluide entre son corps et celui de ses patients, non par l'intervention du Bouddha ${ }^{3}$. Les bouddhistes cinghalais, de

3. Cf. Prothero, 1997, pp. 107-109. 
leur côté, regardaient ces cures comme miraculeuses. Le problème n'est pas, pour nous, de savoir si et pourquoi ils croyaient aux miracles - de toute évidence, ceux-ci faisaient partie de leur horizon mental -, mais de comprendre comment, par quelles voies et suivant quels axes, les exhibitions du Colonel Olcott se constituèrent en manifestations du surnaturel. La situation coloniale y contribua sans doute. Porter l'Américain aux nues revenait à opposer à la domination britannique le pouvoir combien supérieur, parce qu'irrigué par le surnaturel, d'un autre Occidental. Les manifestations du surnaturel peuvent receler, Max Weber l'a souligné, de fortes capacités subversives : quand la religion est parvenue à un degré avancé d'institutionnalisation, s'appuyant sur un groupe stable d'agents dotés d'un charisme de fonction, elles se déroulent, sous leurs formes les plus intenses et entraînantes, à la marge, en dehors du contrôle de ce groupe ; parce qu'elles offrent un espace de légitimation alternatif, elles naissent par ceux qui mettent en question la probité de la prêtrise et récusent son monopole sur les biens de salut (selon la terminologie wébérienne). Au contraire, dans les sociétés marquées par une faible institutionnalisation de la religion, lorsque fait défaut un corps unique et organisé de prêtres, en contexte "animiste » notamment, ces manifestations prolifèrent au cœur de la vie collective et se montrent moins contestataires en termes de rapports sociaux de pouvoir. Cet effet de structure, qui décide de la position, périphérique ou diffuse selon les cas, des expériences du surnaturel et de leur signification confirme à quel point ces expériences sont configurées par des dispositions sociales, autant - si ce n'est plus - que par des dispositions individuelles ( $c f$. les explications psychopathologiques du chamanisme ou de la possession).

Nonobstant leurs différences de position, les manifestations du surnaturel se distinguent par une articulation spécifique avec l'institué, à savoir la tradition (ou la coutume) et le rituel qui en est l'actualisation. Les personnages de médiums qui émergent sur la scène islandaise dans la première moitié du vingtième siècle s'inscrivent dans une histoire longue des relations avec les morts; ils donnent toutefois et une allure inédite au mode de communication avec le monde invisible (en y introduisant une touche chamanique), et un contenu sans précédent aux représentations de ce monde (en le peuplant d'êtres originaux). Aux antipodes, en Birmanie, ont depuis peu surgi, dans les cérémonies du principal culte de possession, des entités qui n'appartiennent pas au panthéon des esprits propre à ce culte ; or, cet avènement a été possible après que les entités en question ont accédé à une incarnation statuaire, laquelle est, au sein du culte, une condition de la possession. D'une part, les manifestations du surnaturel présentent un air familier dans le contexte où elles se déploient; leurs caractères semblent comme contraints par des schémas pré-établis : elles parlent le langage ordinaire de l'extraordinaire. De l'autre, elles n'ont, pour ainsi dire, qu'à moitié le sens des traditions. Elles sont inévitablement marquées de quelque trait insolite, et ce, comme l'attestent les carrières des possédés au Karnataka (sud de l'Inde), parce qu'elles constituent le moyen d'une construction de soi, un procédé d'affirmation d'une individualité ou d'une communauté de destin. Autant les expériences du 
surnaturel découlent d'une médiation collective, autant cette médiation valorise les idiosyncrasies. Les expériences du surnaturel sont le lieu par excellence de l'innovation religieuse.

On s'étonne moins de la relation tourmentée du surnaturel avec le rituel. Celui-ci instaure un régime d'expérience identifiable et normé à travers des logiques de l'action en décalage avec les cadres ordinaires de la pratique - un régime apte à induire le sentiment de l'extraordinaire. Il est des façons de faire et des façons de dire qui ne trompent pas et impartissent à un événement la saveur du surnaturel. Chacun sait alors ce qu'il a à penser, comment se conduire, quoi éprouver. Le rituel se révèle à ce point essentiel à l'expérience du surnaturel que celle-ci se trouve fréquemment enchâssée dans celui-là : chamanisme, possession bénéfique, inspiration divinatoire semblent réservés à des contextes rituels. Les spécialistes des oracles au Japon en savent quelque chose, qui déclarent en substance à l'ethnologue : "Je n'ai pas vraiment besoin de rites, mais les fidèles eux en ont besoin pour être rassurés, pour accepter ce que je dis et fais à ce moment-là ». Mais le surnaturel renâcle à la domestication rituelle, qui a sur lui un effet anesthésiant. Lorsqu'il n'y a plus que du rituel, il n'y a plus d'expérience du surnaturel ainsi de la messe en contexte catholique. Un rituel "scelle ", au double sens de sanctionner et de fixer, une situation : par exemple l'union conjugale de deux individus. Les expériences du surnaturel, pour leur part, fructifient par indétermination. On leur demande de produire de l'insoupçonné ou, au moins, d'exprimer l'indiscernable, l'imprévisible. Elles livrent des réponses à des questions non formulées ou non élucidées. Elles ne consentent pas, à chacune de leurs répétitions, à engendrer un résultat invariable, uniforme par son sens et par sa valeur. Elles se révèlent fluides et réactives, le message qu'elles véhiculent se gonflant des préoccupations les plus actuelles des uns et des autres. L'intervention du surnaturel avivera une crise ou un conflit au sein de la communauté aussi bien qu'elle le résoudra. Les expériences du surnaturel, non conformistes par nature, ouvrent un espace de jeu. Elles font l'histoire autant qu'elles sont faites par l'histoire. Les apparitions de Lourdes se ramènent, en l'espèce, à un cas d'école. En somme, si le rituel est nécessaire à la fabrication d'une expérience du surnaturel, trop de rituel tue cette expérience.

Aussi peut-on avancer cette proposition, troisième et dernière composante de notre argument: les expériences du surnaturel entretiennent avec l'institué un rapport asymptotique, en ce qu'elles épousent ses formes mais déjouent son déterminisme. Si l'on cherchait à tout prix la formule bien frappée, il suffirait de détourner, en l'inversant, la devise du structuralisme triomphant : avec les expériences du surnaturel, plus c'est la même chose, plus ça change.

Voilà quelques balises pour indiquer le spectre de la réflexion. Le propos de ce numéro n'est pas de bâtir du neuf, ni de faire système ou théorie, ni même de défendre une thèse. Il s'agit, au travers d'exemples variés mais sans prétendre à aucune représentativité, de poser un problème intrinsèque aux expériences du surnaturel, problème qu'affrontent depuis toujours, d'une façon ou d'une autre, 
les commentateurs du fait religieux : comment ce qui est pensé et rapporté par les intéressés comme un phénomène anormal, comme un phénomène sujet à caution ou à doute, revêt pourtant in fine l'aspect d'une réalité sociale objective, admise et vécue collectivement, effective ? Ou encore : par quels biais ce qui est défini par les acteurs comme échappant à la perception commune se constituet-il néanmoins sur le mode de l'évidence partagée, s'affirme comme sensible et sensé, indubitable ? Un tel procès de construction s'enracine dans les matériaux idéels propres à la communauté, dans un système de représentations et de valeurs qui dessine un champ de possibles : attribuer une infortune à un esprit implique de tenir pour acquises et l'existence des esprits, et leur façon de s'immiscer dans les affaires humaines. Il se rapporte aussi à une époque et à ses paramètres, il est modulé par des déterminations historiques : de nouvelles entités ne deviennent pas pour rien actives au sein d'un culte à un moment donné. Il tient, encore, à des conditions de fabrication in situ, à tous les discours, objets et pratiques qui fondent un dispositif opérateur de surnaturel. Il est orienté, enfin, par les individus qui y participent et s'en saisissent en l'infléchissant, en y inscrivant leur marque. À considérer ces différents aspects, on embrasse, s'il est faisable, l'ensemble du processus de médiation qui façonne une expérience du surnaturel; et on interroge la croyance, en tant que rapport spécifique et spécifié à une réalité. La tâche est d'autant plus énorme que le surnaturel ne se présente pas sous un aspect unique et homogène au sein d'une société. N'en déplaise au Colonel Olcott, aux côtés du Bouddha et parfois en symbiose avec lui, s'épanouissent maintes divinités, esprits, démons.

Cette manière d'envisager et de sonder les expériences du surnaturel n'interdit pas le recours à des hypothèses psychologiques, d'ailleurs contenues dans les théories des sciences sociales sur la religion. Mais quand l'observateur s'éloigne des éléments tangibles de l'activité collective - pour spéculer par exemple sur les rouages mentaux de l'adhésion de fidèles birmans à l'invraisemblable manifestation en chair et en os de superhommes en principe invisibles - la conclusion apparaît, quoique on pense des vertus heuristiques de la démarche, pure conjecture. Il est vrai qu'en matière d'expériences du surnaturel, les sciences sociales finissent toujours par achopper sur un point quelconque. Heureusement du reste. 